\title{
The contributions of language and experience to the representation of abstract and concrete words: Different weights but similar organizations
}

\author{
J. Frederico Marques • Ludmila D. Nunes
}

Published online: 7 June 2012

(C) Psychonomic Society, Inc. 2012

\begin{abstract}
In the present studies, we evaluated the contributions of language and sensorimotor information to the representation of abstract and concrete words and the possibility that the organizations of the two types of concepts follow different organizational principles: association, for abstract concepts, and semantic similarity, for concrete concepts. In Study 1, we examined the two strongest associates of concrete and abstract words from published free association norms. Study 2 then extended this analysis to individual data collected with a free association task. Language associations were more important for abstract than for concrete words, but for sensorimotor information no differences were observed between the two types of concepts. Also, no clear evidence was found for different qualitative organizational principles for abstract and concrete concepts. Multiple representational systems thus seem to be engaged in the conceptual processing of abstract and concrete concepts, while it remains to be investigated whether their representations follow different organizational principles.
\end{abstract}

Keywords Abstract $\cdot$ Concrete $\cdot$ Language $\cdot$ Sensorimotor information $\cdot$ Free association

In recent years, a diverse set of models regarding conceptual knowledge have proposed that multiple representational systems are engaged in conceptual processing (Andrews, Vigliocco, \& Vinson, 2009; Barsalou, Santos, Simmons, \& Wilson, 2008; Dove, 2009, 2011; Louwerse \& Jeuniaux, 2008, 2010; Simmons, Hamann, Harenski, Hu, \& Barsalou, 2008; Steyvers, 2010). In particular, they have all considered

J. F. Marques $(\bowtie) \cdot$ L. D. Nunes

Faculdade de Psicologia, Alameda da Universidade,

1649-013 Lisboa, Portugal

e-mail: jfmarques@fp.ul.pt that both more sensorimotor-based information and languagebased information play a role in conceptual representation, thus recapturing the ideas first proposed by Paivio (1971, 1986) in the context of the dual-code theory.

This general perspective of the involvement of language and sensorimotor information in conceptual representation is particularly discussed regarding the processing and representation of abstract and concrete concepts. Again, these models agree on a general division of labor in the acquisition of these two types of concepts, with concrete concepts being largely learned through sensorimotor experience, and abstract concepts being largely learned through linguistic experience (Andrews et al., 2009; Crutch \& Warrington, 2005; Dove, 2009, 2011; Louwerse \& Jeuniaux, 2010; Steyvers, 2010). The behavioral data supporting this proposal, however, are still recent and limited. Nevertheless, evidence from both neuropsychological (e.g., Breedin, Saffran, \& Coslett, 1994; Crutch \& Warrington, 2005; Warrington, 1981) and neuroimaging (e.g., Binder, 2007; Sabsevitz, Medler, Seidenberg, \& Binder, 2005) studies converge to show that these two types of concepts differentially activate the brain areas involved in sensorimotor and language representation.

Where the models clearly diverge is on whether this division should also entail different organizational principles in terms of conceptual representation. Most models have assumed that the difference between abstract and concrete concepts is more quantitative, thus emphasizing similar organizational principles (or being neutral as to this question). For instance, Paivio' $\mathrm{s}$ dual-code theory (Paivio, 1986) assumes that concrete words are represented in terms of verbal and nonverbal coding, whereas abstract words only rely on the verbal code, as they lack direct sensory referents. For Schwanenflugel and colleagues (Schwanenflugel, Harnishfeger, \& Stowe, 1988; Schwanenflugel \& Shoben, 1983), concrete words have more related contextual information than do abstract words, whereas 
Plaut and Shallice (1991, 1993) claimed that concrete concepts are supported by a higher number of semantic features. All of these models readily explain the processing advantage of concrete over abstract words in a number of behavioral tasks (e.g., James, 1975; Juhasz \& Rayner, 2003; Paivio, 1971, 1986; Schwanenflugel et al., 1988) and are also in accord with neuroimaging evidence, in terms of both electrophysiological correlates (Kounios \& Holcomb, 1994) and brain activation (Binder, 2007). However, cases in which a reverse concreteness advantage is found are more difficult to accommodate within these perspectives.

This is the case for several reported cases of impairment that affect more concrete rather than more abstract concepts (e.g., Breedin et al., 1994; Crutch, Ridha, \& Warrington, 2006; Crutch \& Warrington, 2005), which has led to the hypothesis that the difference between abstract and concrete concepts is more qualitative. In this context, it has been proposed that different feature types are more important to different concepts (Breedin et al., 1994) or, more fundamentally that, irrespective of the type of information that constitutes abstract and concrete concepts (and of the manner in which they are acquired), a much more central difference exists in the actual architectures of those representations. In particular, concrete concepts are predominantly organized in terms of semantic similarity (i.e., a categorical organization), whereas abstract concepts are predominantly organized by associative links (i.e., an associative network organization) (Crutch et al., 2006; Crutch \& Warrington, 2005). This second idea has received support from evidence with stroke aphasia and deep dyslexic patients (Crutch, 2006; Crutch et al., 2006; Crutch \& Warrington, 2005) tested with spoken word-written word matching tests, and also from analyses of their reading errors. In matching tests, stroke aphasia patients show more errors in the identification of abstract words when the distractors are semantic associates (i.e., thematic-related distractors) rather than nonassociates of the target, but there is no semantic similarity effect (i.e., of direct or close synonyms), whereas for concrete words, more errors are observed for semantically similar distractors (i.e., category coordinates) than for semantically dissimilar distractors, but there is no effect of semantic associates (i.e., thematic-related distractors) (Crutch et al., 2006; Crutch \& Warrington, 2005). However, it should be noticed that while the same type of distractors has been used to evaluate an associative organization in abstract and concrete concepts, different distractors have been used to test the similarity principle. In the case of reading by deep dyslexic patients, more semanticassociative reading errors than synonym reading errors are observed for abstract words, whereas the reverse is observed for concrete words (Crutch, 2006). Recently, a study using spoken word-picture identification with normal participants (Duñabeitia, Avilés, Afonso, Scheepers, \& Carreiras, 2009) also showed that abstract related words activate semantic associated target objects more quickly and strongly (e.g., smell-nose) than do concrete words, thus providing support for this qualitative perspective.

This latter result is important for the generalization of this perspective to the general population, as it could be the case that the pattern of results observed for patients has somehow resulted from particular deficits in semantic processing that had no parallel in normal functioning. However, Duñabeitia et al. (2009) only tested for the prevalence of the semantic association principle for abstract words, but not for the prevalence of the semantic similarity principle for concrete words (this same principle was also not tested with the same materials for abstract and concrete concepts in patients; Crutch et al., 2006; Crutch \& Warrington, 2005). Moreover, all of the targets were concrete objects and, as such, one could argue that their data allow us only to conclude that the association principle seems to govern abstract information related to concrete concepts, but say nothing about the representation of abstract concepts.

In the present studies, we tried to overcome these limitations with a different and more unconstrained task that allowed us to test both of the questions discussed regarding processing and representing abstract and concrete conceptsthe general multiple-representational hypothesis, and the more specific qualitative-versus-quantitative perspective. For this purpose, we considered the single-word association task, in which the participant is presented with a given word and asked to write down or say the first word that comes to mind. This is one of the oldest tasks in psychology, first introduced by Sir Francis Galton (1879) to study naturally occurring associations, which has become extensively used since it was framed in a more experimental approach during the 1950s and 1960s (e.g., Deese, 1962, 1965; Marshall \& Cofer, 1963; Miller \& Glucksberg, 1988; Nelson, McEvoy, \& Dennis, 2000).

Free association ostensibly provides a valid measure of the preexisting strength between a test cue and a related word, meaning that given a particular cue, one response is more likely to occur than another (Nelson et al., 2000). This association strength may reflect different types of relations between concepts, from more collocational, with little or no semantic overlap (e.g., couch-potato, paramour-paramount) to categorical (e.g., robin-bird), or script or thematic relations (e.g., beach-sand) (Chaffin, 1997; Moss \& Older, 1996). As such, generating an associative response may reflect the contributions of our experience with language (Spence \& Owens, 1990) and also of sensorimotor and perceptual experience, as in the case of reading action words that rapidly and automatically activate the motor system in a somatotopic manner (Pulvermüller, 2005). This is also the case in the continuous-association task, in which linguistic information seems to be activated first, and situational and simulation information second (Barsalou et al., 2008; 
Santos, Chaigneau, Simmons, \& Barsalou, 2011; Simmons et al., 2008).

Previous studies of abstract and concrete concepts with this task have seemed to show that concrete words are associated to more quickly than abstract words (de Groot, 1989), and that the number of associates is somewhat larger for abstract words, but only when less reliable idiosyncratic responses are considered (Altarriba, Bauer, \& Benvenuto, 1999; Nelson \& Schreiber, 1992). However, no differences between possible types of associations have been explored in relation to the items' concreteness. Nevertheless, there is also evidence that the type of association produced may be modulated by the word cue characteristics, in a way that reflects the contributions of language and sensorimotor systems. In fact, Chaffin (1997) showed that unfamiliar words elicited more linguistic, nonsemantically based associations than did familiar words, whereas for the latter set more properties of events were produced. As such, considering the two questions put forward for the representation of abstract and concrete concepts, we can also expect that different types of associations may emerge for these two types of concepts.

At a more general level, if multiple representational systems that are engaged in conceptual processing interact with the nature of the items to be processed, we predict that language-based associations will be more important for abstract than for concrete words, whereas experiential and sensorimotor-based associations will be more important for concrete than for abstract words.

Regarding the quantitative-qualitative opposition, if the latter is true we would expect that, independently of the manner in which concepts are acquired, associations reflecting a semantic similarity principle will be more important for concrete than for abstract concepts, whereas associations reflecting semantic association but not semantic similarity will be more important for abstract than for concrete concepts.

We tested these hypotheses in two studies. In the first study, we analyzed the types of association of the strongest associates of concrete and abstract words included in the University of South Florida free association norms (Nelson, McEvoy, \& Schreiber, 2004). The second study replicated and extended the first study through the collection of association responses for the same items and by analyzing the types of association at both the item and subject levels.

\section{Study 1}

In the first study, we selected two groups of abstract and concrete concept words from the Paivio, Yuille, and Madigan (1968) concreteness norms and set out to analyze the types of associations corresponding to their two strongest associates, as different studies have shown that associative strength is inversely related to response reliability to the item (e.g., Altarriba et al.,
1999; Nelson et al., 2000; Nelson \& Schreiber, 1992). The words in the two sets were matched for word familiarity, which also modulates the types of association produced (Chaffin, 1997). Moreover, extended analyses allowed us to take into consideration other differences in set size and associative strength that are common to these sets (e.g., Altarriba et al., 1999; Paivio et al., 1968; Schwanenflugel \& Shoben, 1983).

The coding system for association types was borrowed from Barsalou and colleagues (Barsalou et al., 2008; Santos et al., 2011), as their system allowed for testing our predictions, while not having been developed for that purpose. The system considers three large categories of associations-linguistic (or more language-based), taxonomical, and object-situation or simulation (more sensorimotor-based) - that permitted the evaluation of the general multiple-representational hypothesis. The system also allowed us to consider several subtypes of associations, which allowed for the contrast of associations more reflecting a semantic similarity principle (i.e., synonyms, superordinates, category coordinates, and subordinates) with those more reflecting a semantic association (i.e., thematic or situational associates, or forward and backward completions), independently of their language/sensorimotor nature.

Regarding the first question, if the multiple representational systems engaged in conceptual processing interact with the nature of the item to be processed, we predicted that linguistic associations would be higher for abstract words in comparison with concrete words, while the reverse would be observed for object-situation associations.

Regarding the second question, from a qualitative perspective, we predicted that associations reflecting a semantic similarity principle would be higher for concrete words than for abstract words, whereas for associations reflecting semantic association but not semantic similarity, the reverse would be observed.

\section{Method}

Materials From the Paivio, Yuille, and Madigan (1968) concreteness norms (1-7 rating scale), we first considered all of the nouns that were rated as highly abstract (ratings $<2$ ) and selected all items that were also included in the University of South Florida free association norms (Nelson et al., 2004). Each item selected was paired with a noun rated as highly concrete (ratings $>6.45$; Paivio et al., 1968) included in the same free association norms (Nelson et al., 2004), while controlling for word frequency, familiarity, and length (from Clark \& Paivio, 2004), as well as number of orthographic neighbors. ${ }^{1}$ The total sample of materials included 94 nouns: 47 abstract and 47 concrete (see Table 1

\footnotetext{
${ }^{1}$ Calculated online with the Washington University in St. Louis Speech and Hearing Lab Neighborhood Database: http:/neighborhoodsearch.wustl.edu/ Neighborhood/NeighborHome.asp.
} 
and the Appendix). As is apparent from Table 1, it was not possible to match the two sets in terms of the numbers of associates per word or of set size, $t(92)=3.05, p<.01$. In parallel, as is usually the case (Nelson et al., 2000), words with smaller sets tended to have stronger first associates. For each noun, we selected its two strongest (i.e., first and second) associates from the free association norms (Nelson et al., 2004). In the case of second associates, there were ten ties, for which we randomly selected one of the responses in order to maintain the same proportions of abstract and concrete word cue-response pairs (total $n=188$ ). A $2 \times 2$ ANOVA considering word type (abstract or concrete) and associate (first or second) presented main effects for both variables, which were qualified by a significant interaction, $F(1,184)=6.38, M S E=.08, p<.05$, showing that the abstract and concrete word pairs differed in association strength for their first associates (concrete $>$ abstract), but not their second associates.

Procedure Three independent judges who were blind to the specific hypotheses rated each word cue-response pair in terms of Barsalou and collaborators' procedure (Barsalou et al., 2008; Santos et al., 2011; Simmons et al., 2008). Briefly, they used a hierarchical coding scheme from linguistic to taxonomic to object-situation codings, assuming that although a given association could have different origins, it is statistically more likely for the dimensions to be ordered in this manner, a proposal that has received both behavioral (e.g., Santos et al., 2011) and neuroimaging (Simmons et al., 2008) support.

In more detail, if a response was linguistically related to the word cue, it was coded as such, and consideration of other possible coding categories proceeded no further. The linguistic relations included: forward and backward associations (e.g., $\mathrm{BEE} \Rightarrow$ hive, JACKET $\Rightarrow$ life), synonyms and antonyms (e.g., $\mathrm{CAR} \Rightarrow$ automobile, JUSTICE $\Rightarrow$ injustice), and root and sound similarities (e.g., LAW $\Rightarrow$ lawyer, LAW $\Rightarrow$ straw). If none of these relations applied, the response was evaluated in terms of its possible taxonomic relation to the cue (i.e., superordinate, coordinate, or subordinate). Finally, if a response did not fall into either a linguistic or a taxonomic coding category, it was coded as a response indexing the simulation system. The latter included thematic or situational relations (e.g., FREEDOM $\Rightarrow$ revolution, $\mathrm{CAMEL} \Rightarrow$ desert) and concept properties (e.g., $\mathrm{KNIFE} \Rightarrow$ blade). A last category was considered for responses that did not fit any of the other codes, but no items were included in this category. The interrater agreement at the level of the major categories (linguistic, taxonomic, and simulation) was $95.5 \%$. Disagreements were resolved between judges.

\section{Results and discussion}

To evaluate our first prediction, we considered the total frequencies for the language-based associations and for the sensorimotor-based associations in each concept group (thus, frequencies for taxonomic-based associations were not considered). The results are presented in Fig. 1. Given the nature of the data (i.e., frequencies), nonparametric statistics were employed (chi-square test). An alpha level of $p<.05$ was considered for all analyses.

The pattern of results was in accord with the prediction, although statistical analysis showed a significant difference in the proportions of language-based associations between abstract and concrete concepts $\left[\chi^{2}(1)=5.69, p<.05\right]$, but not in the case of sensorimotor-based associations $\left[\chi^{2}(1)=\right.$ 0.56 , n.s.]. Also, if the analysis was considered for each type of concept, the language-based associations were significantly higher than the sensorimotor-based associations for abstract concepts $\left[\chi^{2}(1)=9.67, p<.05\right]$, but this difference was nonsignificant for concrete concepts $\left[\chi^{2}(1)=0.00\right.$, n.s.]. As such, the results seem to show that language associations are more important for abstract than for concrete concepts, while sensorimotor associations seem equally important for both types of concepts.

To evaluate our second prediction, we considered the total frequencies for the associates reflecting a semantic similarity principle or categorical organization (Crutch, 2006; Crutch et al., 2006; Crutch \& Warrington, 2005), including synonyms, superordinates, category coordinates, and subordinates. In a similar vein, we considered all of the associates reflecting a semantic association principle but not semantic similarity - that is, "all associate words whose meanings are not synonymous but which are often bound together in real world or sentential contexts" (Crutch \& Warrington, 2005, p. 622) —which corresponded to thematic or situational associates and forward and backward completions. Frequencies of the two types of associates by concept groups are presented in Fig. 2.

In this case, the pattern of results was just the opposite of what would be expected from a qualitative perspective, with

Table 1 Stimulus characteristics

\begin{tabular}{lllllll}
\hline & Concreteness & Familiarity & Word Frequency & Length & Number Orthographic Neighbors & Set Size \\
\hline Abstract words $(n=47)$ & 1.70 & 6.45 & 1.48 & 6.38 & 3.53 & 17.35 \\
Concrete words $(n=47)$ & 6.79 & 6.39 & 1.55 & 6.30 & 2.89 & 14.45 \\
\hline
\end{tabular}




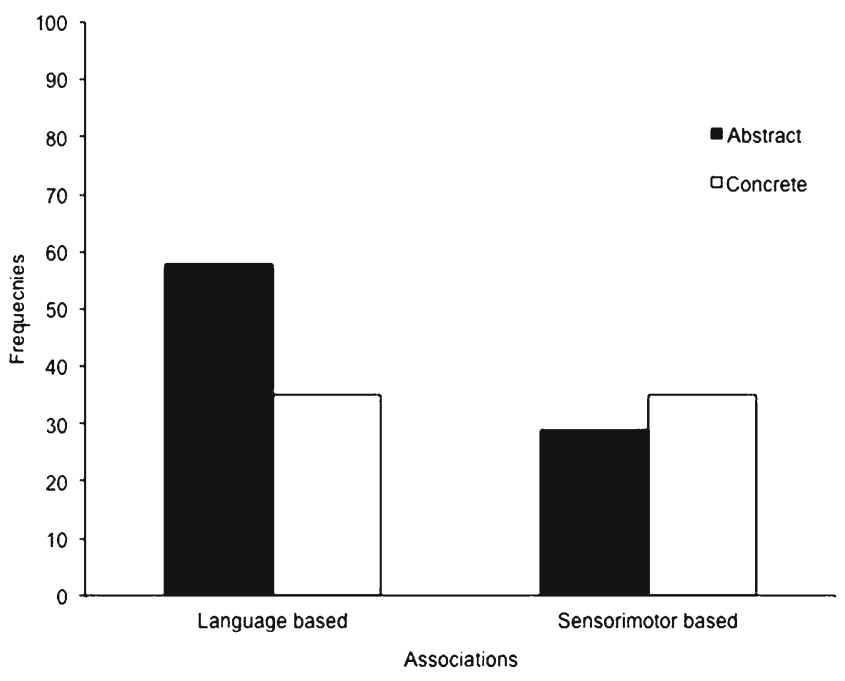

Fig. 1 Language-based and sensorimotor-based associations for abstract and concrete concepts (Study 1)

more semantic similarity associations for abstract than for concrete concepts $\left[\chi^{2}(1)=4.35, p<.05\right]$, and the reverse observed for semantic associations, although this result was not significant $\left[\chi^{2}(1)=2.59\right.$, n.s.]. Also, if the analysis was considered for each type of concept, semantic associations were significantly higher than the semantic similarity associations for concrete concepts $\left[\chi^{2}(1)=4.35, p<.05\right]$, but this difference is nonsignificant for abstract concepts $\left[\chi^{2}(1)=2.59\right.$, n.s. $]$.

To further evaluate whether this pattern of results was related to differences between abstract and concrete sets at the level of associative strength or number of associates, we ran a binary logistic regression considering concreteness, associative strength, and number of associates as independent variables and type of association (linguistic or sensorimotor) as a dependent variable. The regression with the three

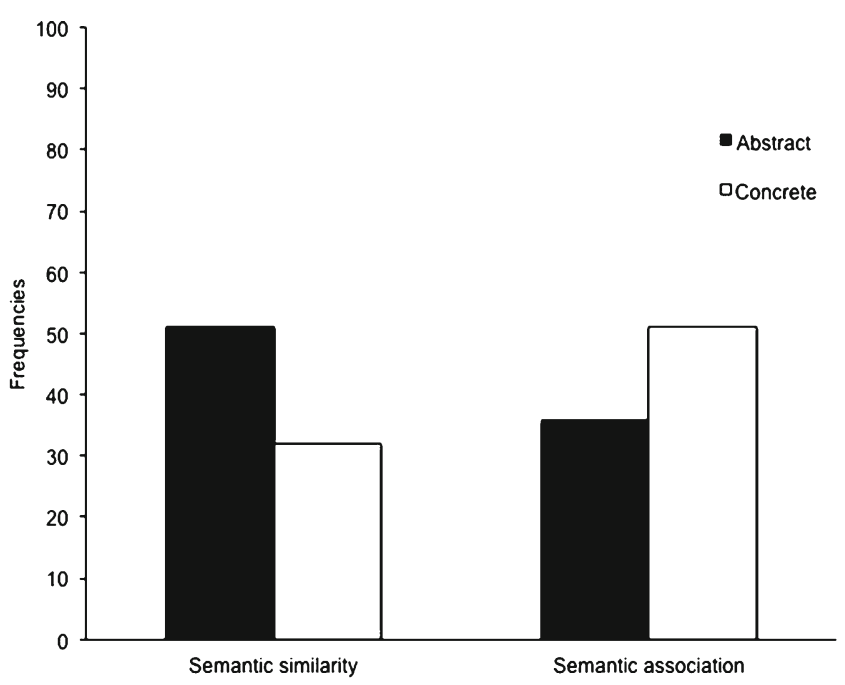

Fig. 2 Associates reflecting semantic similarity and semantic association for abstract and concrete concepts (Study 1) variables yielded an overall nonsignificant model $\left[\chi^{2}(2)=\right.$ $6.65, p=.57]$, but one in which concreteness $(b=-.18$, $S E=.07$, Wald $=7.10, p<.05)$, unlike associative strength $(b=.58, S E=1.24$, Wald $=0.22, p=.64)$ or number of associates $(b=.001, S E=.04$, Wald $=0.06, p=.81)$, was individually predictive of the type of association. These results thus show that the imbalance between abstract and concrete sets for these dimensions had no impact in terms of the types of associates produced.

A final important aspect of the results was that associates reflecting synonym associations were particularly preponderant for abstract concepts, constituting $76 \%$ of the language-based associations registered in the first analysis and $86 \%$ of the similarity-based associations in the second analysis. The impact of this particular type of association was further explored in Study 2.

\section{Study 2}

In this study, we replicated and extended Study 1 by collecting association responses for the same items and by analyzing the types of associations at both the item (similarly to Study 1) and subject levels. This last aspect was very important, as association norms provide an array of responses for a population of individuals that may or may not be representative of the associative structure of particular individuals (Nelson et al., 2000; Osipow \& Grooms, 1965). As such, it is important to evaluate whether the same results can be observed when we look at the individual level and at more "idiosyncratic" responses that are usually discarded from association norms.

\section{Method}

Participants A group of 35 native English speakers, with a mean age of 20 years and with an undergraduate education, voluntarily participated in this experiment.

Materials The materials used were the same as in Study 1.

Procedure The 94 nouns were presented on a computer screen, one by one and in random order, using E-Prime software. The participants were tested individually and were informed that they would be presented with a list of nouns, one at a time, and that their task would be to type in for each noun the first word that came to their mind that was meaningfully related or strongly associated to the presented noun. The participants were also informed that there were no right or wrong answers and that it was very important to type in the first word each presented noun made them think of. Each noun was presented at the center of the computer screen, and the participants had no time limit to answer. After entering an answer and pressing Enter, a new noun was presented on 
the screen. At the end of the task, the participants were thanked and debriefed.

The participants' answers were analyzed, and the words produced in response to each presented noun were ranked, according to their absolute and relative frequencies of production. The misspelled words were eliminated. All of the presented nouns were then coupled to all of the produced answers, forming a total of 3,277 word cue-response pairs (and 1,711 different word-cue pairs, after collapsing across repetitions).

As in Study 1, three independent judges who were blind to the specific hypotheses rated each word cue-response pair by considering Barsalou and collaborators' (Barsalou et al., 2008; Simmons et al., 2008) procedure. The interrater agreement at the level of the major categories (linguistic, taxonomic, and simulation) was $94 \%$. Disagreements were resolved between the judges.

\section{Results and discussion}

Similarly to Study 1 , for the analysis by items, ${ }^{2}$ we considered the total frequencies for the language-based (or linguistic) associations and for the sensorimotor-based (or simulation) associations in each concept group for the two strongest associates of each word (again, in the case of ties, we randomly selected one of the responses in order to maintain the same proportions of abstract and concrete word cue-response pairs; $n=186$ ). These results are presented in Fig. 3.

The results showed a pattern of associations for the two concepts similar to the one in Study 1: Statistical analysis confirmed a significant difference in the proportions of language-based associations between abstract and concrete concepts $\left[\chi^{2}(1)=5.15, p<.05\right]$, but not in the case of

\footnotetext{
${ }^{2}$ As in Study 1, the number of associates observed was higher for abstract than for concrete concepts $[t(92)=4.09, p<.05]$. Moreover, a $2 \times 2$ ANOVA considering word type (abstract or concrete) and associate (first or second) revealed main effects for both variables that were qualified by a significant interaction, $F(1,184)=4.22, M S E=.06$, $p<.05$, showing that abstract and concrete word pairs differed in association strength for first associates (concrete $>$ abstract) but not for second associates. Nevertheless, similarly to Study 1, a separate ANOVA considering concept group and the two types of ratings as independent variables and associative strength and number of associates as dependent variables only showed significant main effects for concept group, $F(1,156)=5.88, M S E=.14, p<.05$ (for Ratings 1), and $F(1,159)=5.55, M S E=.13, p<.05$ (for Ratings 2), in the case of associative strength, and $F(1,156)=35.06, M S E=812.70, p<.05$ (for Ratings 1), and $F(1,159)=27.54, M S E=602.4, p<.05$ (for Ratings 2 ), in the case of number of associates. Again, these results confirm an imbalance between abstract and concrete sets for these dimensions but show that these differences had no impact in terms of the type of associates produced.
}

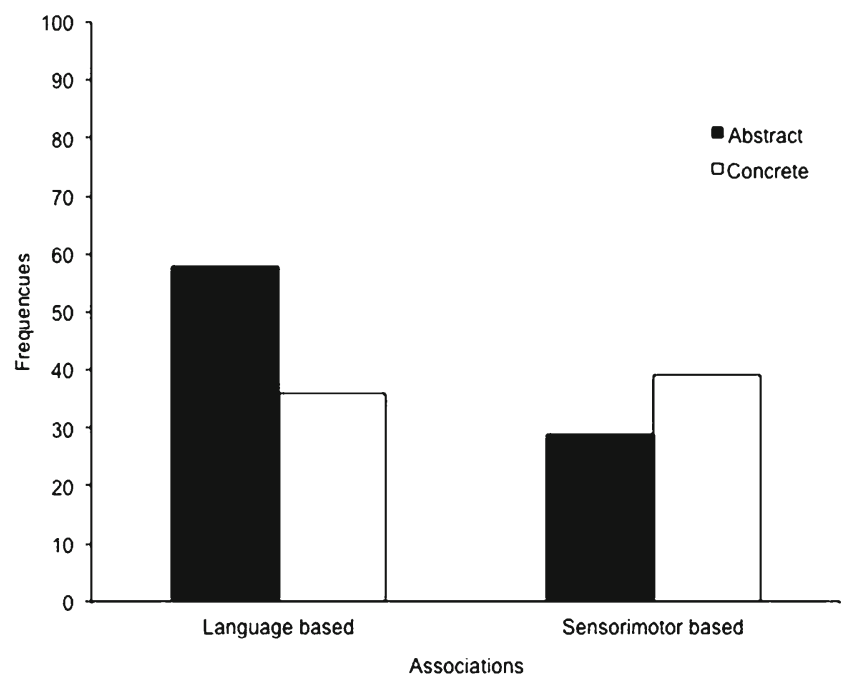

Fig. 3 Language-based and sensorimotor-based associations for abstract and concrete concepts (Study 2)

sensorimotor-based associations $\left[\chi^{2}(1)=1.47\right.$, n.s. $]$. Again, when we compared the two types of associations for each concept type, the difference was significant for abstract concepts $\left[\chi^{2}(1)=9.67, p<.05\right]$ but not for concrete concepts $\left[\chi^{2}(1)=0.22\right.$, n.s. $]$. The results thus replicated Study 1 , showing that language associations are more important for abstract than for concrete concepts, while sensorimotor associations seem equally important for both types of concepts.

We also calculated the percentages of the different types of associations by subjects, separately for abstract and concrete concepts (considering all items). The mean percentages (and standard deviations) of language-based and sensorimotor-based associations for abstract and concrete concepts (by subjects) are presented in Table 2 .

Statistical analysis ( $t$ tests for dependent samples) again showed that the proportion of language-based associations was higher for abstract words than for concrete words $[t(34)=3.30, p<.05]$, but there was no significant difference in the case of sensorimotor-based associations $[t(34)=1.33$, n.s.], which again confirmed the same pattern of results on the individual level. Nevertheless, when we compared the two types of associations for each concept type, sensorimotorbased associations were significantly higher than languagebased associations for both concrete $[t(34)=3.30, p<.05]$ and abstract $[t(34)=2.09, p<.05]$ concepts, which was due to this analysis considering all of the associations, and not only the first and second strongest associates.

To evaluate our second prediction, we considered at the item level the total frequencies for the associates reflecting a semantic similarity principle (i.e., synonyms, superordinates, or category coordinates and subordinates) and for the associates reflecting a semantic association principle but not semantic similarity (i.e., thematic or situational associates, or forward and backward completions) in each 
Table 2 Mean percentages (and standard deviations) of language-based and sensorimotor-based associations for abstract and concrete concepts

\begin{tabular}{lll}
\hline & Language-Based Associations & Sensorimotor-Based Associations \\
\hline Concrete & $36.61(7.77)$ & $46.57(8.34)$ \\
Abstract & $42.73(8.45)$ & $48.96(9.65)$ \\
\hline
\end{tabular}

concept group and for the two strongest associates of each word (Fig. 4).

As in Study 1, the pattern of results was just the opposite of what would be expected from a qualitative perspective, with more semantic similarity associations for abstract than for concrete concepts $\left[\chi^{2}(1)=6.05, p<.05\right]$, and the reverse observed for semantic associations, although the results was not significant $\left[\chi^{2}(1)=0.98\right.$, n.s. $]$. Again, when we compared the two types of associations for each concept type, the difference was significant for concrete concepts, showing an advantage of semantic associations $\left[\chi^{2}(1)=3.85\right.$, $p<.05]$, but not for abstract concepts $\left[\chi^{2}(1)=2.23\right.$, n.s. $]$.

We also calculated the percentages of associates reflecting semantic similarity and semantic association by subjects, separately for abstract and concrete concepts. The mean percentages (and standard deviations) of associates reflecting semantic similarity and semantic association for abstract and concrete concepts (by subjects) are presented in Table 3.

Statistical analysis ( $t$ tests for dependent samples) showed that the proportion of semantic similarity-based associates was higher for abstract than for concrete concepts $[t(34)=9.30, p<.05]$, while exactly the reverse pattern was observed for semantic-based associates $[t(34)=9.30$, $p<.05]$. Moreover, when we compared the two types of associations for each concept type, the difference was significantly favorable for semantic associations over semantically similar associations for both abstract $[t(34)=8.90$,

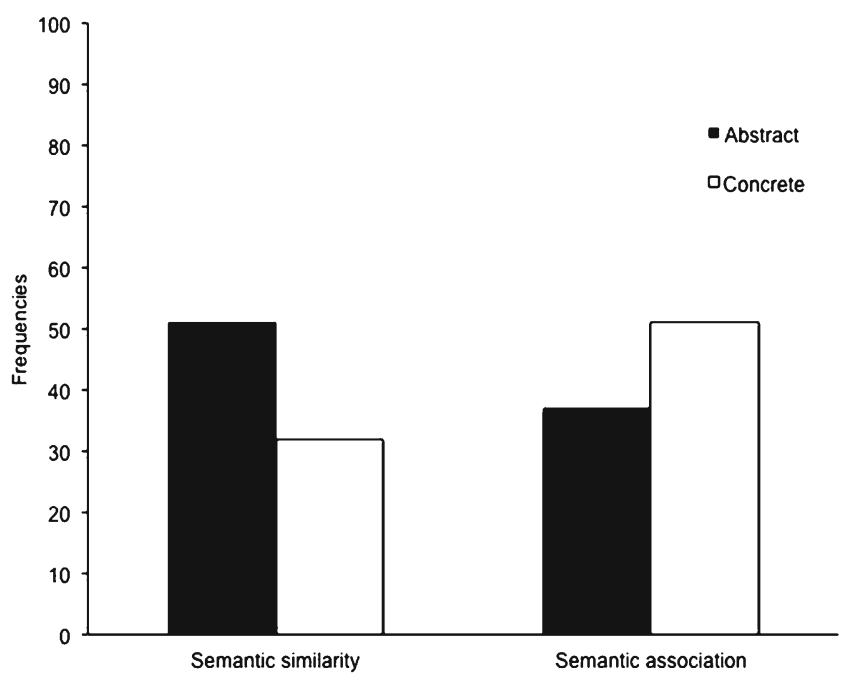

Fig. 4 Associates reflecting semantic similarity and semantic association for abstract and concrete concepts (Study 2) $p<.05]$ and concrete $[t(34)=20.09, p<.05]$ concepts. These results, which allowed for an evaluation of the associative structures of particular individuals, thus confirmed the same negative results for the similarity principle obtained from more global data (both Studies 1 and 2), but they give some support for a semantic-association-based organization for abstract concepts. However, this result was weakened by the fact that semantic associates were also more important for concrete concepts, and by the fact that the results for the strongest associates in both studies failed to show the expected advantage of this principle for abstract concepts.

As in Study 1, associates reflecting synonym associations were particularly preponderant for abstract concepts, constituting $75 \%$ of the language-based associations registered in the first analysis and $100 \%$ of the similarity-based associations in the second analysis when we considered only the two strongest associates. When all responses were considered (as in the analysis by subjects), the impact of synonym relations for abstract concepts significantly decreased (to $45 \%$ in the first analysis and $65 \%$ in the second analysis), while the general pattern of results was similar to the more global analysis by items. Nevertheless, the results from both studies highlight the importance of this particular type of relation to abstract concepts, which we will further examine in the General Discussion.

\section{General discussion}

In the present article, we set out to answer two questions regarding the processing and representation of abstract and concrete concepts. First, considering more recent proposals that multiple representational systems are engaged in conceptual processing (e.g., Andrews et al., 2009; Barsalou et al., 2008; Dove, 2009, 2011; Louwerse \& Jeuniaux, 2008, 2010; Steyvers, 2010), we evaluated whether language information

Table 3 Mean percentages (and standard deviations) of associates reflecting semantic similarity and semantic association for abstract and concrete concepts

\begin{tabular}{lll} 
& Semantic Similarity & Semantic Association \\
\hline Concrete & $22.49(8.10)$ & $77.51(8.10)$ \\
Abstract & $35.02(9.95)$ & $64.98(9.95)$ \\
\hline
\end{tabular}


would be more important for the representation of abstract words, while experiential and sensorimotor information would be more important for concrete words. Second, we also evaluated whether abstract words would predominantly activate semantically associated concepts, whereas concrete words would predominantly activate semantically similar concepts. Importantly, in both cases this evaluation was conducted while controlling for familiarity, linguistic frequency, and length, all of which have impacts on word recognition (e.g., Balota, Cortese, Sergent-Marshall, Spieler, \& Yap, 2004) and word association (Chaffin, 1997). Moreover, extended analysis showed that the number of associates and the associative strength, which also influence word recognition (Duñabeitia, Avilés, \& Carreiras, 2008), did not impact on the type of association produced.

Regarding the first question, the results from our two studies clearly indicate that language associations are more important for abstract concepts than for concrete concepts (and more important than sensorimotor information for abstract concepts), while no difference for sensorimotor information was found between the two types of concepts (nor between language information for concrete concepts). These results thus support the preponderant role attributed to language for the acquisition and representation of abstract concepts, while for concrete concepts both types of information, language and sensorimotor, seem equally important. These results are in general agreement with a dual-code perspective (Paivio, 1986), in support of the idea that concrete words are equally represented in terms of verbal and nonverbal coding, whereas abstract words rely more on the verbal code. Importantly, however, the fact that sensorimotor information seems similarly relevant for the representation of abstract and concrete concepts (or even more important than language-based information in both types of concepts in the Study 2 analysis by subjects) does not conform to the dual-code perspective. In contrast, results seem to point out that even abstract concepts have sensorimotor referents and may be embodied in sensory experience (e.g., Barsalou \& Wiemer-Hastings, 2005; Glenberg \& Kaschak, 2002). The fact that what is here is classified as sensorimotor also includes event-based information (this type of association was more recently referred to by Santos et al., 2011, as object or situation descriptor and would generally correspond to what Chaffin, 1997, classified as event-based associations) cannot serve as a defense of the dual-code perspective, as in any case these associations are equally nonverbal. On the contrary, the fact that they include event information is an additional argument for the importance of sensory experience and simulation for the representation of abstract concepts (Barsalou \& Wiemer-Hastings, 2005).

Regarding the second question, the present results don't seem to support either the claim that concrete words especially activate semantically similar concepts or the claim that abstract words especially activate semantically associated concepts. On the contrary, the results from both studies seem to show that semantically similar associates are more activated for abstract concepts than for concrete concepts and are also less activated than semantic associates for this last instance. When all answers are considered at the individual level (by-subjects analysis) in Study 2, there is evidence that abstract concepts activate more semantically associated concepts than semantically similar concepts. However, the same pattern of results is observed for concrete concepts and, moreover, both studies fail to find an advantage of semantic associates over semantically similar associates for the two strongest associates.

The present results are not incongruent with Duñabeitia et al. (2009), as they only tested for the activation of semantic associates and particularly analyzed speed of activation for abstract information related to concrete concepts. Nevertheless, the present results are not in agreement with Crutch and colleagues' results (Crutch, 2006; Crutch et al., 2006; Crutch \& Warrington, 2005), where it is the prevalence of the two types of associates (semantic and similar) that was tested. It is possible that the task used in the present study was more biased to activating semantic associates than to activating semantically similar concepts and, as such, prevented the manifestation of the qualitative differences between abstract and concrete word representations (the results from the by-subjects analysis in Study 2 are in accord with this idea). However, it is also important to note that previous studies with patients did not directly contrast semantic similarity with semantic association, but only each organization with a nonorganized set (Crutch et al., 2006; Crutch \& Warrington, 2005). As such, these results may have overestimated the role of each principle for the representation of each type of concept.

In this context, it is also important to discuss the particular case of the synonym relations that were predominant for abstract concepts and, as such, are driving an important part of the reported effects. In fact, their assignment as language-based associates (e.g., Santos et al., 2011) and also as associates reflecting "semantic similarity" (e.g., Crutch et al., 2006) may not be an obvious choice, although we think it is completely justified. In the first case, all other language association types, with exception of antonyms, seem to have a clearer "linguistic nature," reflecting either co-occurrence in text or speech (i.e., forward/backward associations) or phonological/orthographic relations (i.e., root and sound similarities). However, what is at stake in this taxonomy is the fact that language associations more probably reflect an acquisition grounded on language, while what we designate as sensorimotor associations more probably reflect an acquisition grounded on sensorimotor experience (Barsalou et al., 2008), and this is also the reason why we chose it to evaluate the multiplerepresentational-systems hypothesis. As such, in this context both synonyms and antonyms are more probably learned through language than through experience. In 
accordance with this position, synonyms and at least some antonyms are said to not only define semantic relations, but also lexical relations, occurring together often in texts, and also being related by other aspects such as morphological form or collocational patterns (Murphy, 2010). In the case of semantic similarity, Crutch and associates (Crutch et al., 2006; Crutch \& Warrington, 2005) considered that synonyms, similar to category coordinates, superordinates, or subordinates, reflect the categorical relations between concepts that are all based on this principle. Interestingly, all of these relations are also grouped together by Chaffin (1997) in what he called "definitional associations"- that is, relations in which the associate tries to define the meaning of the cue. Moreover, he also opposed this type of association to what he called "event-based associations," which largely correspond to Crutch's semantic-association principle (Chaffin, 1997).

Again, what we think these results show is that a more qualitative difference in the representation of abstract and concrete concepts based on the relative importances of semantic similarity and semantic association at best is only warranted for this last principle. For the activation of semantic associates by abstract concepts, there is in fact some support from both patient studies (Crutch, 2006; Crutch et al., 2006; Crutch \& Warrington, 2005) and studies with healthy participants (Duñabeitia et al., 2009, and this study), although all suffer from particular limitations that call for further testing with other designs and tasks and a more diverse set of measures. In this context, is also important to consider the continuous nature of concreteness and what consequences should be expected in terms of representation for the certainly large number of concepts that these studies have not yet evaluated. Another possible avenue for research is to consider how the differences between the two types of concepts evolve over time (e.g., through a continuous-association task). If Santos et al. (2011) are correct, we would expect that some of these differences could in fact become attenuated, given that situational associations are expected to be predominant over time. Nevertheless, the interaction of time and concept concreteness remains here an empirical question to be addressed.

In conclusion, it seems clear that multiple representational systems are needed to account for the conceptual processing of abstract and concrete conceptsnamely, language and sensorimotor systems. Whether the representation of these concepts also implies fundamental different organizational principles remains an issue to be investigated.

Author note We thank David Kellen and K. Andrew DeSoto for their help collecting the data and for their useful comments.

\section{Appendix}

Table 4 List of words used in Study 1 and presented as targets in Study 2

\begin{tabular}{|c|c|c|c|}
\hline \multicolumn{2}{|c|}{ Abstract Concepts } & \multicolumn{2}{|c|}{ Concrete Concepts } \\
\hline Anger & Jealousy & Barrel & Nursery \\
\hline Anxiety & Joy & Bird & Peach \\
\hline Attitude & Kindness & Bottle & Pencil \\
\hline Belief & Knowledge & Bouquet & Potato \\
\hline Blessing & Love & Cellar & Priest \\
\hline Bravery & Loyalty & Claw & Prisoner \\
\hline Chance & Memory & Clock & Proprietor \\
\hline Concept & Mood & Corner & Pudding \\
\hline Confidence & Moral & Cradle & Railroad \\
\hline Criterion & Nonsense & Dress & Restaurant \\
\hline Deceit & Obsession & Fire & Rock \\
\hline Democracy & Passion & Flower & Rod \\
\hline Ego & Prestige & Forehead & Salad \\
\hline Essence & Pride & Grass & Spinach \\
\hline Fate & Shame & House & Storm \\
\hline Freedom & Soul & Hurricane & Strawberry \\
\hline Glory & Spirit & Juggler & Ticket \\
\hline Greed & Tendency & Lake & Toast \\
\hline Grief & Theory & Machine & Umbrella \\
\hline Happiness & Thought & Magazine & Valley \\
\hline Honour & Truth & Maiden & Vehicle \\
\hline Hope & Vanity & Microscope & Volcano \\
\hline Idea & Virtue & Mountain & Wine \\
\hline Intellect & & Nail & \\
\hline
\end{tabular}

\section{References}

Altarriba, J., Bauer, L. M., \& Benvenuto, C. (1999). Concreteness, context availability, and imageability ratings and word associations for abstract, concrete, and emotion words. Behavior Research Methods, Instruments, \& Computers, 31, 578-602.

Andrews, M., Vigliocco, G., \& Vinson, D. (2009). Integrating experiential and distributional data to learn semantic representations. Psychological Review, 116, 463-498.

Balota, D. A., Cortese, M. J., Sergent-Marshall, S. D., Spieler, D. H., \& Yap, M. J. (2004). Visual word recognition for single-syllable words. Journal of Experimental Psychology. General, 133, 283316. doi:10.1037/0096-3445.133.2.283

Barsalou, L. W., Santos, A., Simmons, W. K., \& Wilson, C. D. (2008). Language and simulation in conceptual processing. In M. de Vega, A. M. Glenberg, \& A. C. Graesser (Eds.), Symbols, embodiment, and meaning (pp. 245-283). Oxford: Oxford University Press.

Barsalou, L. W., \& Wiemer-Hastings, K. (2005). Situating abstract concepts. In D. Pecher \& R. Zwaan (Eds.), Grounding cognition: The role of perception and action in memory, language, and thought (pp. 129-163). Cambridge: Cambridge University Press.

Binder, J. R. (2007). Effects of word imageability on semantic access: Neuroimaging studies. In J. Hart Jr. \& M. A. Kraut (Eds.), Neural 
basis of semantic memory (pp. 149-180). Cambridge: Cambridge University Press.

Breedin, S. D., Saffran, E. M., \& Coslett, H. B. (1994). Reversal of the concreteness effect in a patient with semantic dementia. Cognitive Neuropsychology, 11, 617-660. doi:10.1080/02643299408251987

Chaffin, R. (1997). Associations to unfamiliar words: Learning the meanings of new words. Memory and Cognition, 25, 203-226.

Clark, J. M., \& Paivio, A. (2004). Extensions of the Paivio, Yuille, and Madigan (1968) norms. Behavior Research Methods, Instruments, \& Computers, 36, 371-383. doi:10.3758/BF03195584

Crutch, S. J. (2006). Qualitatively different semantic representations for abstract and concrete words: Further evidence from the semantic reading errors of deep dyslexic patients. Neurocase, 12, 91-97.

Crutch, S. J., Ridha, B. H., \& Warrington, E. K. (2006). The different frameworks underlying abstract and concrete knowledge: Evidence from a bilingual patient with a semantic refractory access dysphasia. Neurocase, 12, 151-163.

Crutch, S. J., \& Warrington, E. K. (2005). Abstract and concrete concepts have structurally different representational frameworks. Brain, 128, 615-627.

de Groot, A. M. (1989). Representational aspects of word imageability and word frequency as assessed through word association. Journal of Experimental Psychology: Learning, Memory, and Cognition, 15, 824-845. doi:10.1037/0278-7393.15.5.824

Deese, J. (1962). On the structure of associative meaning. Psychological Review, 69, 161-175. doi:10.1037/h0045842

Deese, J. (1965). The structure of associations in language and thought. Baltimore: Johns Hopkins Press.

Dove, G. O. (2009). Beyond perceptual symbols: A call for representational pluralism. Cognition, 110, 412-431.

Dove, G. O. (2011). On the need for embodied and dis-embodied cognition. Frontiers in Cognition, 1, 242. doi:10.3389/fpsyg.2010.00242

Duñabeitia, J. A., Avilés, A., Afonso, O., Scheepers, C., \& Carreiras, M. (2009). Qualitative differences in the representation of abstract versus concrete words: Evidence from the visual-word paradigm. Cognition, 110, 284-292. doi:10.1016/j.cognition.2008.11.012

Duñabeitia, J. A., Avilés, A., \& Carreiras, M. (2008). NoA’s Ark: Influence of the number of associates in visual word recognition. Psychonomic Bulletin and Review, 15, 1072-1077. doi:10.3758/PBR.15.6.1072

Galton, F. (1879). Psychometric experiments. Brain, 2, 149-162.

Glenberg, A. M., \& Kaschak, M. P. (2002). Grounding language in action. Psychonomic Bulletin and Review, 9, 558-565. doi:10.3758/BF03196313

James, C. T. (1975). The role of semantic information in lexical decisions. Journal of Experimental Psychology: Human Perception and Performance, 104, 130-136.

Juhasz, B. J., \& Rayner, K. (2003). Investigating the effects of a set of intercorrelated variables on eye fixation durations in reading. Journal of Experimental Psychology: Learning, Memory, and Cognition, 29, 1312-1318.

Kounios, J., \& Holcomb, P. J. (1994). Concreteness effects in semantic processing: ERP evidence supporting dual-coding theory. Journal of Experimental Psychology: Learning, Memory, and Cognition, 20, 804-823.

Louwerse, M. M., \& Jeuniaux, P. (2008). Language comprehension is both embodied and symbolic. In M. de Vega, A. Glenberg, \& A. C. Graesser (Eds.), Symbols, embodiment, and meaning (pp. 309326). Oxford: Oxford University Press.

Louwerse, M. M., \& Jeuniaux, P. (2010). The linguistic and embodied nature of conceptual processing. Cognition, 114, 96-104.

Marshall, G. R., \& Cofer, C. N. (1963). Associative indices as measures of word relatedness: A summary and comparison of ten methods. Journal of Verbal Learning and Verbal Behavior, 1, 408-421.
Miller, G. A., \& Glucksberg, S. (1988). Psycholinguistics aspects of pragmatics and semantics. In R. C. Atkinson, R. J. Herrnstein, G. Lindzey, \& R. D. Luce (Eds.), Stevens' handbook of experimental psychology (2nd ed., Vol. 2, pp. 417-472). New York: Wiley.

Moss, H. E., \& Older, L. (1996). Birkbeck word association norms. Hove: Psychology Press.

Murphy, M. L. (2010). Lexical meaning. Cambridge: Cambridge University Press.

Nelson, D. L., McEvoy, C. L., \& Dennis, S. (2000). What is free association and what does it measure? Memory and Cognition, 28, 887-899. doi:10.3758/BF03209337

Nelson, D. L., McEvoy, C. L., \& Schreiber, T. A. (2004). The University of South Florida free association, rhyme, and word fragment norms. Behavior Research Methods, Instruments, \& Computers, 36, 402-407. doi:10.3758/BF03195588

Nelson, D. L., \& Schreiber, T. A. (1992). Word concreteness and word structure as independent determinants of recall. Journal of Memory and Language, 31, 237-260.

Osipow, S. H., \& Grooms, R. R. (1965). Comparisons between cultural and individual associative response hierarchies. Journal of Verbal Learning and Verbal Behavior, 4, 94-97.

Paivio, A. (1971). Imagery and verbal processes. New York: Holt, Rinehart and Winston.

Paivio, A. (1986). Mental representations: A dual coding approach. Oxford: Oxford University Press.

Paivio, A., Yuille, J. C., \& Madigan, S. A. (1968). Concreteness, imagery and meaningfulness values for 925 nouns. Journal of Experimental Psychology, 76(1, Pt. 2), 1-25. doi:10.1037/ h0025327

Plaut, D. C., \& Shallice, T. (1991). Effects of word abstractness in a connectionist model of deep dyslexia. In Proceedings of the 13th Annual Meeting of the Cognitive Science Society (pp. 73-78). Hillsdale, NJ: Erlbaum.

Plaut, D. C., \& Shallice, T. (1993). Deep dyslexia: A case study of connectionist neuropsychology. Cognitive Neuropsychology, 10, $377-500$.

Pulvermüller, F. (2005). Brain mechanisms linking language and action. Nature Reviews Neuroscience, 6, 576-582.

Sabsevitz, D. S., Medler, D. A., Seidenberg, M., \& Binder, J. R. (2005). Modulation of the semantic system by word imageability. NeuroImage, 27, 188-200.

Santos, A., Chaigneau, S. E., Simmons, W. K., \& Barsalou, L. W. (2011). Property generation reflects word association and situated simulation. Language and Cognition, 3, 83-119.

Schwanenflugel, P. J., Harnishfeger, K. K., \& Stowe, R. W. (1988). Context availability and lexical decisions for abstract and concrete words. Journal of Memory and Language, 27, 499-520.

Schwanenflugel, P. J., \& Shoben, E. J. (1983). Differential context effects in the comprehension of abstract and concrete verbal materials. Journal of Experimental Psychology: Learning, Memory, and Cognition, 9, 82-102. doi:10.1037/0278-7393.9.1.82

Simmons, W. K., Hamann, S. B., Harenski, C. N., Hu, X. P., \& Barsalou, L. W. (2008). fMRI evidence for word association and situated simulation in conceptual processing. The Journal of Physiology, 102, 106-119.

Spence, D. P., \& Owens, K. C. (1990). Lexical co-occurrence and association strength. Journal of Psycholinguistic Research, 19, $317-330$

Steyvers, M. (2010). Combining feature norms and text data with topic models. Acta Psychologica, 133, 234-243. doi:10.1016/ j.actpsy.2009.10.010

Warrington, E. K. (1981). Concrete word dyslexia. British Journal of Psychology, 72, 175-196. 